\title{
An automated open-field apparatus utilizing an improved resistance detection circuit
}

\author{
MORRIS K. MORGRET \\ Washington State University, Pullmah, Washington 99163 \\ and \\ PAUL R. ALBEE \\ Stanford Research Institute, Menlo Park, California 94025
}

\begin{abstract}
An open-field apparatus that can be automatically operated is described. Detection of movement is electronic and based upon the fact that the $S$ will complete a circuit path when crossing an open field. The circuit is very sensitive yet quite immune to the usual problem of $60-\mathrm{Hz}$ interference.
\end{abstract}

The open field has a long and extensive history in the field of experimental psychology, and it continues to be in use today (see Joffe \& Levine, 1973; Tamasy, Koranyi, Lissak, \& Jandala, 1973; Nash, 1973). However, it is a somewhat primitive technique since it involves observation during which many problems may arise: E bias, accuracy, problems of vigilance over extended periods, limited duration of observation, etc. Because of these problems, an automated field was developed to provide the necessary data without the problems introduced by the presence of a human $\mathrm{O}$.

\section{APPARATUS}

The basic concept arose from the fact that the resistance of an experimental $\mathrm{S}$ can be detected by a pircuit that is sensitive to the presence of a high resistance across two poles. To have the necessary contacts, an open field was constructed in which the squares were of a conductive material. For the prototype model, 4-1/8 $\times$ 4-1/8 in. pieces were cut from thin stainless steel stock. An electrical contact was soldered to the back of the metal and each piece contact cemented to a piece of $1 / 4-\mathrm{in}$. Masonite of the same size. Twenty-five of these squares were arranged on a flat floor with a space $1 / 4$ in. wide between each piece. The $v_{4}$-in. spaces were filled with pieces of Plexiglas 10 provide insulation between the conducting squares. The Plexiglas pieces were rectangular across their top and were cemented with epoxy paint which also served to fill and waterproof the surface. The Plexiglas pieces extended $1 / 8 \mathrm{in}$. above the stainless steel surface. This arrangement of the Plexiglas insulating strips was chosen to eliminate short circuits due to urination. Any liquid placed upon the strips forms a puddle on the floor and a pead along the top of the Plexiglas strip. The vertical edge of the Plexiglas does not allow the surface tension of urine to form a continuous contact between the floor and the top of the strip. This arrangement effectively eliminates the possibility of urine shorting two contacts, but the strip is not high enough to impede movements of he Ss.
The squares are electrically connected, as shown in Fig. 1. The "checker boarding" was chosen as the most efficient and effective arrangement of contact plates. Any contact made by the $S$ between an " $A$ " and " $B$ " contact activates the circuit.

The animal's movements are detected in both the horizontal and vertical directions. Movements made on a strictly diagonal place are not detected. This arrangement appears to allow for some error in missed counts, but this virtually never occurs with mice or rats. Observations confirm the fact that almost invariably an $\mathrm{S}$ will step on an adjacent square when moving in a diagonal direction.

To complete the field, sides were added and the joint between the floor and side was sealed and made water tight. As an added precaution, the entire field was shielded by a covering of copper window screen.

\section{DETECTION CIRCUIT}

Two operational amplifiers are required for the

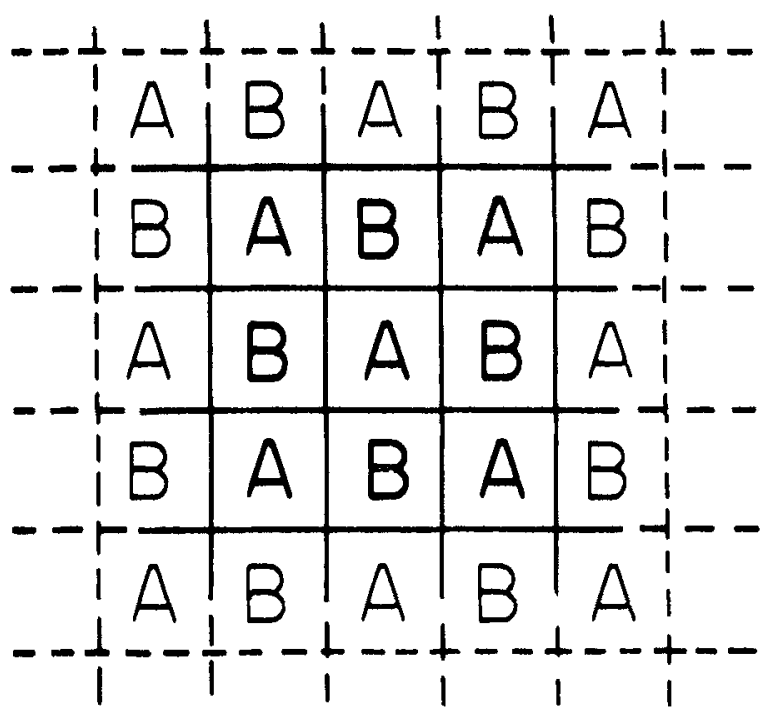

Fig. 1. Arrangement of open field squares. 


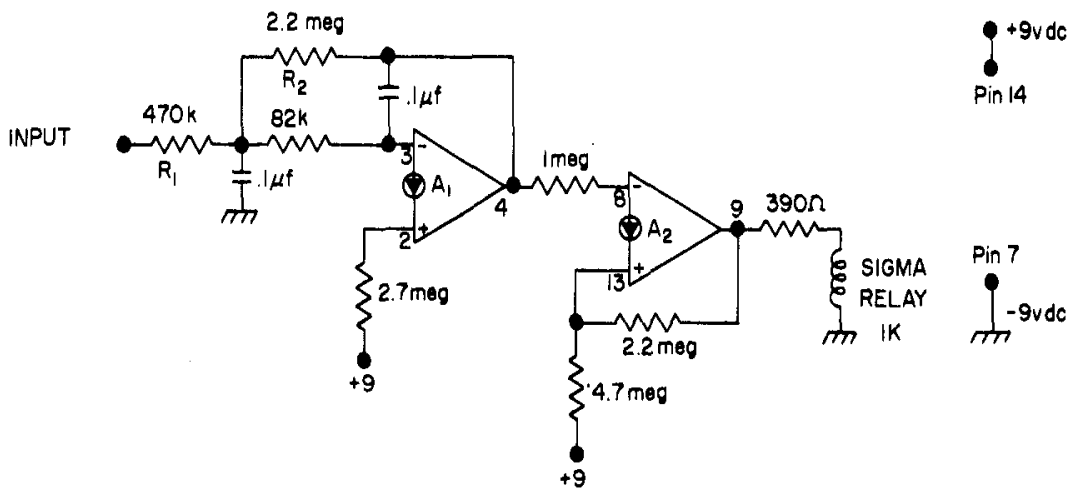

Fig. 2. Schematic of detector circuit. Parts list: $R_{1}=470 \mathrm{~K} ; R_{2}=2.2$ megohm; $R_{3}=82 \mathrm{~K} ; R_{4}=2.7 \mathrm{megohm} ; R_{5}=$ 1 megohm; $R_{6}=4.7$ megohm; $R_{7}=$ $2.2 \mathrm{megohm} ; \mathbf{R}_{\mathbf{s}}=390 \mathrm{ohm} ; \mathrm{C}_{1}=$ $.1 \mathrm{microF} ; \mathrm{C}_{2}=.1$ microF; $\mathrm{IC}_{1}=\mathrm{LM} 3900$, National Semiconductors; $R L_{1}=$ Sigma Relay, 1K ohm.

circuit. The National Semiconductors device LM 3900 was chosen since it is of very low cost and contains four of the needed operational amplifiers. The resistance sensor schematic diagram is shown in Fig. 2.

Amplifier $A_{1}$ is connected as an inverting active low-pass filter with dc gain $=4.7$, and cutoff frequency $=$ $3.75 \mathrm{~Hz}$. Rejection of any spurious $60-\mathrm{Hz}$ signals picked up by the input wiring should be about $50 \mathrm{~dB}$ relative tc the dc signal. Amplifier $A_{2}$ is connected as an inverting Schmidt trigger circuit which drives the $1 \mathrm{k}$ ohm Sigma Relay directly. Current is limited to $6 \mathrm{~mA}$ by the series $390-$ ohm resistor. The Schmidt trigger trip points and input circuit gain were selected so that the relay "pulls in" with an input voltage of $1.6 \mathrm{~V}$ (at $2.3 \mu \mathrm{A}$ ) and releases when the input voltage falls below $0.8 \mathrm{~V}(0.6 \mu \mathrm{A})$. Trip-point sensitivity is dependent on the ratio $R_{2} / R_{1}$, so that increasing $R_{1}$ by a factor of two reduces the sensitivity by a factor of two. $R_{2}$ is one of the components that determines the low-pass cutoff frequency; thus, $R_{2}$ should not be changed when only a gain change is desired (see Tobey, Graeme, \& Huelsman, 1971).

Pin connections are shown for the amplifiers and for $\mathrm{dc}$ power to the quad device. Duplication of this circuit performance using discrete components requires at least six transistors plus four additional resistors and one diode.

To connect the circuit to the open field, all " $A$ " squares are interconnected and then connected to the positive terminal of the power supply. All "B" squares are interconnected and attached to the input of the sensor. Any shielding should be led to the ground of the sensor circuit. Since the $60-\mathrm{Hz}$ rejection is approximately $50 \mathrm{~dB}$, shielding is not mandatory but highly recommended as a precaution against spurious signals. The relay output of the detector may be connected to electromechanical counters or converted to logic levels to interface with logic recording systems. Some logic systems can be driven directly from the output of Amplifier $A_{2}$. In our application, the relay closure operates a pulse former whose output is divided by a clock timer and stepper to count in four equal time segments.

The automated open-field apparatus offers several advantages over observational techniques: It is automated so that data collection is simpler and very complex sequences of observation are possible; it is quite inexpensive and relatively simple to construct; it is reliable and rules out the possible contamination effects of having an $\mathrm{O}$ present; and it is more sensitive to movement than the usual observational procedures. Parts for the electronic circuit should cost less than $\$ 10$.

\section{REFERENCES}

Joffe, J. M. \& Levine, S. Effects of weaning age and adult $h$ and ling on avoidance conditioning, open field behavior, and plasma corticosterone of adult rats. Behavioral Biology, 1973, 9, 235-244.

Nash, D. J. Influence on genotype and neonatal irradiation upon open-field locomotion and elimination in mice. Journal of Comparative \& Physiological Psychology, 1973, 83, 458-464. Tamasy, V., Koranyi, L., Lissak, K., \& Jandala, M. Open-field behavior, habituation and passive avoidance learning: Effect of ACTH and hydrocortisone on normal and adrenalectomized rats. Physiology \& Behavior, 1973, 10, 995-1000.

Tobey, G. E., Graeme, J. G., \& Huelsman, L. P. (Eds.), Operational amplifiers design and applications. New York: McGraw-Hill, 1971.

(Received for publication January 18, 1974; revision received February $21,1974$. ) 